\title{
Efeito dos diferentes intervalos de recuperação na percepção subjetiva de homens e mulheres
}

http://dx.doi.org/10.11606/1807-5509201700040759

\author{
Iara Corsini ALVES* \\ Thamiris Martins NASCIMENTO* \\ Juliana da Silva MENDONÇA* \\ Jeberson Carlos do CARMO* \\ André Calil e SILVA** \\ Cíntia Duarte da SILVA*/**** \\ Sandro Fernandes da SILVA* \\ *Grupo de Estudo e \\ Pesquisa em Respostas \\ Neuromusculares \\ (GEPREN), \\ Departamento de \\ Educação Física, \\ Universidade Federal \\ de Lavras, Lavras, MG, \\ Brasil. \\ **Faculdade de Educação \\ Física e Desportos da \\ Universidade Federal \\ de Juiz de Fora; Juiz de \\ Fora, MG, Brasil. \\ ***Faculdade \\ Presbiteriana GAMMON \\ Lavras, MG, Brasil.
}

\section{Resumo}

0 tempo de intervalo entre as séries no exercício físico é uma variável que vem sendo utilizada como um meio de controle da intensidade e densidade do treinamento. Assim, o objetivo do estudo foi verificar a Percepção Subjetiva Esforço (PSE) em intervalos de recuperação (IRs) de 1 e 3 minutos durante 3 séries consecutivas no aparelho Leg Press $45^{\circ}$ até a falha concêntrica momentânea entre homens e mulheres, utilizando-se da escala de OMNI-RES. Fizeram parte do estudo 14 voluntários, sendo 7 homens ( 22 anos $\pm 3,14 ; 78 \pm 11,42 \mathrm{Kg}$; $1,74 \mathrm{~m} \pm 0,05$ ) e 7 mulheres ( 23 anos $\pm 3,76 ; 56,4 \mathrm{Kg} \pm 6,88 ; 1,63 \mathrm{~m} \pm 0,07)$, ambos fisicamente ativos. Os dados foram coletados em dias alternados e de forma aleatorizada, respeitando um intervalo de 48 horas para realização da coleta da PSE. Para comprovar a distribuição da amostra foi utilizado o teste de Shapiro Wilk. Para identificar as diferenças entre o número de repetições e a PSE entre os gêneros foi adotado o teste de Mann-Whitney. Os resultados mostraram que na comparação entre os gêneros nas variáveis PSE, número de repetições e a soma do número de repetições nos dois intervalos estudados, não foi possivel identificar diferença significativa entre os gêneros. Houve uma diminuição no número de repetições nos dois IRs, porém, não houve diferença significativa na PSE entre as séries em ambos os intervalos. A utilização da mesma para controle de intensidade para homens e mulheres, ainda é questionável, necessitando de mais estudos acerca do tema.

PalavRAS-Chave: Percepção Subjetiva de Esforço; Intervalos de Recuperação; Número de Repetições; Diferença entre Gêneros.

\section{Introdução}

Nos últimos anos o treinamento resistido (TR) tornou-se amplamente conhecido devido a sua capacidade de ampliar a força muscular, bem como melhorar a aptidão física de crianças, adultos e idosos $^{1,2}$. A força muscular recebe o respaldo da comunidade científica por propiciar aos praticantes do treinamento resistido a manutenção da saúde, da habilidade funcional e da qualidade de vida ${ }^{3}$.

Para a prescrição dos exercícios resistidos (ER) uma série de variáveis deve ser controlada, podendose destacar o intervalo entre séries e sessóes, a frequência semanal, o número de repetiçóes e séries e as intensidades das cargas de trabalho ${ }^{4}$. Porém, entre todas essas supracitadas, os intervalos de recuperação entre séries têm-se demonstrado uma variável de suma importância a ser manipulada para nortear as adaptaçôes decorrentes do treinamento ${ }^{5-7}$. No que diz respeito aos intervalos de recuperação entre séries, ainda existe uma escassez de dados sobre essa formatação ${ }^{8}$. BotTARO et al. ${ }^{9}$ identificaram o intervalo entre séries de 30 segundos como sendo o mais efetivo para avaliação do pico de torque em cadeira isocinética, por outro lado, PARCELl et al. ${ }^{10}$ demonstraram que 90 segundos seria o intervalo ideal para a retomada do exercício, 
tentando esclarecer a dúvida sobre o intervalo de recuperação ERNESTO et al. ${ }^{11}$ sugerem que o intervalo entre séries deve ser o suficiente para não influenciar negativamente o volume de treino e esses intervalos deveriam ser de 60 a 180 segundos, dependendo do nível de condicionamento do indivíduo.

Uma variável que pode auxiliar no controle da intensidade do esforço produzido pelos exercícios resistidos é a percepção subjetiva de esforço (PSE). Estudos realizados por BorG ${ }^{12}$ a partir da década de 50 , propuseram através de uma escala, obter o esforço percebido de sujeitos pedalando em cicloergômetros, e verificaram que a tensão percebida durante o exercício aumentava linearmente de acordo com a intensidade, dessa maneira, originou-se o conceito de $\mathrm{PSE}^{12}$. Porém a escala validada por Borg ${ }^{12}$ tinha como finalidade obter a percepção do esforço apenas em exercícios com características aeróbicas, contudo, ao longo dos anos diferentes escalas foram desenvolvidas a fim de se verificar a ação da PSE ao se relacionar as diversas variáveis do treinamento de força. RoBERTSON et al. ${ }^{13}$ elaboraram e validaram a escala OMNI-RES (OMNIResistance Exercise Scale), composta por figuras ilustrativas com levantamento de peso, com a finalidade de associação com o esforço percebido pelo indivíduo quando realizado o exercício resistido.

\section{Método}

\section{Amostra}

A amostra foi constituída por 14 voluntários, 7 homens ( $22 \pm 3.14$ anos; $78 \pm 11.42$ quilos; $1.74 \pm 0.05$ metros; $13 \pm 6.02 \%$ de gordura) e 7 mulheres ( $23 \pm 3.76$ anos; $56 \pm 6.88$ quilos; $1.63 \pm 0.07$ metros; $18,40 \pm 3.79 \%$ de gordura). As descrições das características da amostra estão expostas na TABELA 1. As coletas foram realizadas no Laboratório de Estudos do Movimento Humano - LEMOH, da Universidade Federal de Lavras - UFLA, tendo os seguintes critérios de inclusão: (a) indivíduos fisicamente ativos em treinamento resistido há pelo menos 6 meses, (b) ausência de patologias cardiovasculares e (c) ausência de problemas osteomioarticulares que impedissem a realização do teste. Os participantes do experimento assinaram o termo de consentimento livre e esclarecido (TCLE), segundo a resoluçáo do Conselho Nacional de Saúde (CNS - 196/96). Além disso, os sujeitos foram orientados a não praticar exercícios exaustivos e
A PSE, principalmente após a validação da escala OMNI-RES sem dúvida tem se mostrado um excelente método de controle do treinamento, porém é necessário ressaltarmos as possíveis diferenças nas respostas da mesma encontrada entre os gêneros. BARQUILHA et al. ${ }^{14}$ demonstraram que para uma carga de $70 \%$ do RM, homens e mulheres apresentam resultados semelhantes em relação ao desempenho no volume de repetiçóes. HunTER $^{15}$ esclarece que as mulheres parecem resistir melhor a fadiga que os homens, fatores esses que veem sendo estudados pela comunidade científica. $\mathrm{Na}$ literatura encontram-se variados estudos que investigaram o comportamento de diferentes intervalos de recuperação (IRs) no número de repetiçóes das séries subsequentes ${ }^{5,6,16-18}$, outros avaliaram aspectos como a resposta metabólica, a concentração de GH e de cortisol ${ }^{7,19}$, mas todos esses estudos avaliaram uma única população, exclusivamente homens, assim, na literatura ainda faltam estudos que comparem os gêneros utilizando açōes musculares dinâmicas, o volume de repetições e a PSE.

Dessa maneira, o objetivo do presente estudo foi verificar a PSE através da escala OMNI-RES e o volume de repetiçóes em IRs de 1 e 3 minutos, durante 3 séries consecutivas no aparelho de Leg Press $45^{\circ}$ entre homens e mulheres fisicamente ativos.

que não ingerissem bebidas alcoólicas por um período de 24 horas que antecedessem os testes.

\section{Procedimentos}

\section{A: Avaliaçáo antropométrica}

A estatura e a massa corporal foram medidas em uma balança com estadiômetro (Marca Welmy -Modelo $110 \mathrm{CH})$. Os indivíduos foram posicionados sobre a plataforma da balança de forma ereta, com os pés juntos, as mãos posicionadas ao lado do corpo e a cabeça fazendo um ângulo de $90^{\circ}$ com o pescoço. Para a mensuraçáo do percentual de gordura foi utilizado o protocolo de 3 dobras cutâneas de JACKSON e POLLOCK ${ }^{20}$ para homens e mulheres, sendo que para os homens foram coletadas as dobras torácica, abdominal e do terço médio da coxa e para mulheres foram coletadas as dobras tricipital, suprailíaca e do terço médio da coxa. 
As medidas da espessura das dobras cutâneas foram feitas com o auxílio de um compasso (Cescorf - modelo tradicional). Aferiram-se as medidas das dobras cutâneas sempre do lado direito dos avaliados e com os mesmos em posição anatômica. Os sujeitos vestiam biquíni e sunga, ou uma vestimenta que náo pressionasse e nem cobrisse nenhum um ponto de medição.

\section{B: Teste de carga máxima (1RM)}

Os testes de 1RM foram realizados no aparelho de Leg Press 45 (LP) (Physicus - Modelo PRO15), utilizando anilhas de 5, 10, 15 e $20 \mathrm{~kg}$. Para obtenção da carga, os indivíduos chegaram 15 minutos antes da realização do teste para aquecimento prévio ${ }^{21}$. Os avaliados primeiramente realizaram um aquecimento geral de 10 minutos em uma esteira ergométrica a $4 \mathrm{Km} / \mathrm{h}$ com uma inclinaçáo de $0 \%$, logo após os mesmos realizaram um alongamento e um aquecimento específico no próprio aparelho com um ritmo de execução de $2 / 2$, sendo 2 segundos de execução para a fase concêntrica e 2 segundos de execução para a fase excêntrica do movimento, ao final os mesmos executaram 15 repetiçóes como é proposto por Chagas et al..$^{22}$ e Haskell et al. ${ }^{23}$

$\mathrm{O}$ teste foi realizado conforme as recomendaçóes de BAECHLE e EARLE ${ }^{24}$, com a carga máxima de 1RM obtida entre uma e cinco tentativas. $\mathrm{Na}$ execução do aparelho LP, o indivíduo ficou sentado com o dorso totalmente apoiado no banco e em um ângulo de $45^{\circ}$, pernas paralelas, com um pequeno afastamento lateral e joelhos flexionados, braços ao longo do corpo segurando a barra de apoio. A partir desse momento, os sujeitos deveriam desbloquear a barra de apoio, e realizar uma extensão do quadril e do joelho com flexão plantar e flexionar o joelho a no máximo $90^{\circ}$ controlados através de um goniômetro digital (Miotec ${ }^{\oplus}$ - modelo GN360), em seguida, mover a plataforma realizando ao final do movimento uma extensão completa dos joelhos e do quadril.

\section{C: Processo de coletas}

O processo de coleta ocorreu em 6 visitas realizadas no laboratório de musculaçáo da Universidade Federal de Lavras - UFLA. Nas 3 primeiras visitas realizadas com intervalo de 48 horas entre elas, foram realizadas o processo de familiarização com o teste de $1 \mathrm{RM}$, a partir da $4^{\mathrm{a}}$ visita os indivíduos foram submetidos a uma avaliação antropométrica e em seguida foi realizada a determinaçáo de 1RM de cada voluntário, a partir de então estabeleceu-se um intervalo de 48 horas para o início das séries de execução. $\mathrm{Na} 5^{\mathrm{a}}$ visita as coletas foram feitas de forma aleatória, ou seja, ao realizar a execução de 3 séries consecutivas no exercício de LP com a carga de $80 \%$ de $1 \mathrm{RM}$, uma parte dos indivíduos realizava o teste com um intervalo de 1 minuto e a outra com um intervalo de 3 minutos. $\mathrm{Na}^{\mathrm{a}}$ visita os intervalos entre as séries foram realizados de acordo com a $5^{\mathrm{a}}$ visita, aqueles que anteriormente utilizaram o intervalo de 1 minuto, realizaram o teste com o intervalo de 3 minutos, e os que realizaram com 3 minutos anteriormente, utilizaram 1 minuto na terceira visita. $\mathrm{Na} 5^{\mathrm{a}}$ e na $6^{\mathrm{a}}$ visita, para realização das repetições das séries do exercício os sujeitos foram orientados a executar o máximo de repetiçôes possíveis em cada uma das séries, até que os mesmos não conseguissem vencer a resistência oferecida configurando uma incapacidade funcional. Para que não houvesse interferência na percepção de cada um, as coletas foram realizadas individualmente. É devidamente importante ressaltar que foi afixado um pôster contendo a escala OMNI-RES de frente ao aparelho de LP, a fim de facilitar a visualizaçáo dos participantes durante a execução do exercício.

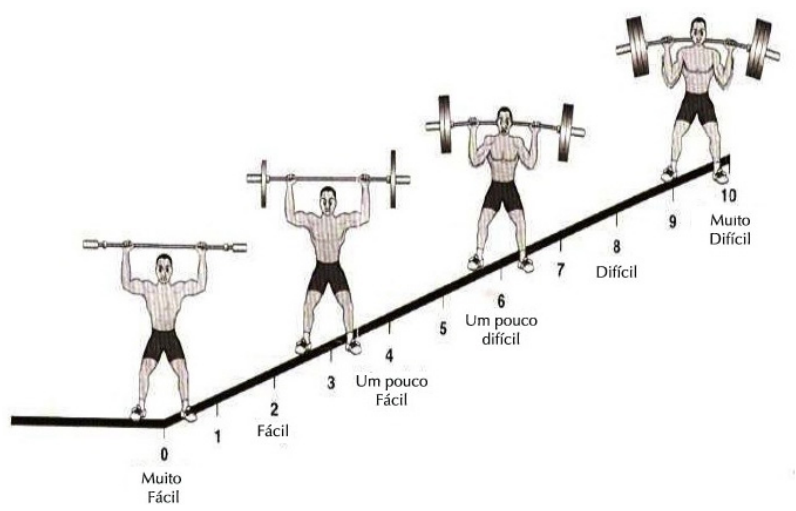

FIGURA 1 - Escala OMNI-RES para exercícios resistidos em adultos ${ }^{13}$. 


\section{D: Tratamento estatístico}

Foi realizada uma análise estatística descritiva com comparação de médias e desvio padrão. Foi utilizado o teste de Shapiro Wilk para verificar a distribuição da amostra. Para identificar as diferenças entre o número de repetiçóes e a PSE entre os gêneros em cada intervalo foi adotado o teste de Mann-Whitney.
Para verificar as PSE e número de repetiçóes em cada intervalo na análise intragêneros foi utilizado o Teste Anova de Medidas Repetidas com o post Hock de Bonferroni para comprovação estatística. Para a variável soma do número de repetiçôes na análise intragêneros foi utilizado o teste não paramétrico de Wilcoxon. Para comprovação estatística foi adotado o $\mathrm{p}<0,05$.

\section{Resultados}

Nas tabelas 1 e 2 demonstramos os resultados da comparação entre os gêneros e intragêneros na variável PSE nos dois intervalos estudados. Foi possível identificar que não existe diferença entre os gêneros na PSE. Já na análise intragêneros, verificamos diferença significativa no gênero masculino no intervalo de 1 minuto entre a $1^{\text {a }} \mathrm{e}$ $3^{\text {a }}$ série, enquanto que no intervalo de 3 minutos as diferenças foram entre a $1^{\mathrm{a}} \times 2^{\mathrm{a}} ; 1^{\mathrm{a}} \times 3^{\mathrm{a}}$; e $2^{a} \times 3^{a}$. No gênero feminino para o intervalo de 1 minuto destacamos uma diferença significativa entre a $1^{a} \times 3^{a}$ série, enquanto que no intervalo de 3 minutos a diferença foi novamente entre a $1^{a} \times 3^{a}$ série.

TABELA 1 - Características da amostra.

\begin{tabular}{cccc}
\hline \multirow{2}{*}{ Intervalo } & Séries & Masculino & Feminino \\
\cline { 2 - 4 } & & $7,29 \pm 1,11^{\mathrm{a}}$ & $7,86 \pm 1,34^{\mathrm{b}}$ \\
\cline { 2 - 4 } 1 & 2 & $8,43 \pm 0,97$ & $8,29 \pm 0,95$ \\
\cline { 2 - 4 } & 3 & $9,29 \pm 0,48$ & $9,57 \pm 0,53$ \\
\hline \multirow{2}{*}{3} & 1 & $7,71 \pm 0,75^{\mathrm{c}, \mathrm{d}}$ & $8,43 \pm 1,81^{\mathrm{f}}$ \\
\hline & 2 & $8,57 \pm 0,53^{\mathrm{e}}$ & $9,97 \pm 53$ \\
\hline
\end{tabular}

a) Existe diferença no grupo masculino na PSE entre a $1^{\mathrm{a}}$ e $3^{\mathrm{a}}$ série no intervalo de 1 minuto;

b) Existe diferença no grupo feminino na PSE entre a $1^{\mathrm{a}}$ e $3^{\mathrm{a}}$ série no intervalo de 1 minuto;

c) Existe diferença no grupo masculino na PSE entre a $1^{\mathrm{a}}$ e $2^{\mathrm{a}}$ série no intervalo de 3 minutos;

d) Existe diferença no grupo masculino na PSE entre a $1^{\mathrm{a}}$ e $3^{\mathrm{a}}$ série no intervalo de 3 minutos;

e) Existe diferença no grupo masculino na PSE entre a $2^{\mathrm{a}}$ e $3^{\mathrm{a}}$ série no intervalo de 3 minutos;

f) Existe diferença no grupo feminino na PSE entre a $1^{\mathrm{a}} \mathrm{e} 3^{\mathrm{a}}$ série no intervalo de 3 minutos.
$\mathrm{Na}$ TABELA 2 apresentamos os resultados do número de repetiçóes por série e a soma das repetiçốes nos intervalos estudados. Não existiu diferença significativa entre os gêneros na análise das variáveis número de repetições e soma das repetições nos 2 intervalos estudados. No gênero masculino houve diferença significativa entre o número de repetições na $3^{\circ}$ série e na soma das repetições entre os intervalos de 1 e 3 minutos. No gênero feminino a tendência se manteve semelhante ao masculino tendo diferença significativa entre o número de repetiçóes na $3^{\circ}$ série e na soma das repetiçóes entre os intervalos de 1 e 3 minutos. 
TABELA 2 - Número de repetições por série e soma das repetições nos intervalos.

\begin{tabular}{|c|c|c|c|}
\hline \multirow{2}{*}{ Intervalo } & \multirow{2}{*}{ Séries } & \multicolumn{2}{|c|}{ No de Repetiçóes Gênero } \\
\hline & & Masculino & Feminino \\
\hline \multirow{4}{*}{1} & 1 & $12,43 \pm 4,31$ & $10,86 \pm 3,28$ \\
\hline & 2 & $10,43 \pm 2,14$ & $8,43 \pm 3,25$ \\
\hline & 3 & $8,86 \pm 1,86^{\mathrm{a}}$ & $7,29 \pm 2,98^{c}$ \\
\hline & Soma Reps & $31,71 \pm 3,90^{\mathrm{b}}$ & $26,57 \pm 8,69^{\mathrm{d}}$ \\
\hline \multirow{4}{*}{3} & 1 & $13,86 \pm 3,93$ & $11,29 \pm 4,19$ \\
\hline & 2 & $11,29 \pm 2,05$ & $9,71 \pm 2,21$ \\
\hline & 3 & $10,43 \pm 2,22$ & $9,43 \pm 2,44$ \\
\hline & Soma Reps & $35,57 \pm 6,02$ & $30,43 \pm 7,78$ \\
\hline
\end{tabular}

a) Existe diferença significativa no grupo masculino no $\mathrm{n}^{\circ}$ repetiçóes entre os intervalos de 1 minutos e 3 minutos na $3^{\text {a }}$ serie;

b) Existe diferença entre a soma de repetições no grupo masculino entre os intervalos de 1 e 3 minutos; c) Existe diferença significativa no grupo feminino no $\mathrm{n}^{\circ}$ repetiçôes entre os intervalos de 1 minutos e 3 minutos na $3^{\text {a }}$ serie;

d) Existe diferença significativa no grupo feminino na soma do o $\mathrm{n}^{\circ}$ repetiçôes na comparação entre os intervalos (1 x 3).

\section{Discussão}

Os principais resultados deste trabalho demonstram que o intervalo de recuperação tem o efeito semelhante na realização do exercício tanto em homens quanto em mulheres, diminuindo o número de repetições na última série para ambos os gêneros. Os resultados sugerem ainda, que não há diferença na PSE no exercício de Leg Press $45^{\circ}$ nos dois grupos amostrais, para as diferentes séries e intervalos de recuperação.

IDE et al. ${ }^{4}$ têm proposto que o intervalo de recuperação entre séries de exercícios resistidos determinam a magnitude da formação dos estoques de energia fosfagênica (ATP-CP) e da glicólise anaeróbia, interferindo significativamente nas respostas metabólicas, hormonais e cardiovasculares durante o treinamento. Além disso, a extensão do IR influencia na remoção dos metabólitos produzidos na contração muscular e contribui para a diminuição da fadiga muscular ${ }^{1}$. Desta forma, quanto menores os intervalos de recuperação, maiores serão os níveis de fadiga ${ }^{1}$, independentemente do número de repetições ${ }^{25}$.

A American College of Sports Medicine ${ }^{3}$ denota que, para praticantes intermediários $\mathrm{e}$ avançados do treinamento resistido sáo recomendados intervalos de recuperação entre as séries de 2 a 3 minutos ao realizar exercícios multiarticulares. De acordo com Kraemer e HäKKIneN ${ }^{26}$, os intervalos de recuperação entre as séries no treinamento de força devem ser longos para que ocorra uma melhor recuperação do sistema nervoso e energético, assim o indivíduo será capaz de suportar a mesma carga nas próximas séries do exercício.

O presente trabalho corrobora com os estudos de Willardson e Burkett ${ }^{6}$ e Ratamess et al. ${ }^{7}$ onde demonstraram que intervalos de recuperação menores que 3 minutos promovem uma diminuição no volume total (carga $\mathrm{x}$ repetiçóes), ou seja, quando comparados IRs de 1 minuto ao IRs de 3 minutos, os referidos estudos apresentaram uma diminuição no número de repetições das séries subsequentes, bem como o encontrado em nosso trabalho, que a soma das repetições foram menores significativamente entre os IRs de 1 minutos e IRs de 3 minutos. Ainda nessa linha e apresentando resultados semelhantes SIMÃo et al. ${ }^{27}$ investigaram diferentes intervalos de recuperação ( 2 e 5 minutos) para a realização de três séries para 10RM no exercício supino horizontal e voador frontal, onde pôde-se perceber uma redução significativa no número de repetições, de acordo com a evolução das séries. Nesse estudo, os resultados mostraram que mesmo havendo diminuição de repetiçóes nos dois exercícios, pôde-se verificar que o 
exercício supino foi mais susceptível a fadiga, mesmo utilizando um intervalo maior de recuperação. Semelhante ao nosso estudo, o exercício de Leg Press $45^{\circ}$ também é um exercício multiarticular, independente do gênero (homens e mulheres), podemos relacionar a diminuição de repetiçóes entre as séries com a fadiga causada aos músculos auxiliares no movimento realizado.

Os resultados do nosso estudo mostraram que mesmo havendo uma diminuição significativa no número de repetições na última série utilizando-se de 2 IRs, constatou-se que houve diferença significativa na PSE entre os mesmos. Resultados dispares aos nossos, foram encontrados por Woods et al. ${ }^{28}$ onde foram examinados os efeitos de diferentes tempos de intervalo sobre a PSE durante 3 séries de 10 repetiçóes na $\mathrm{CE}$ a $70 \%$ de $10 \mathrm{RM}$, com intervalos entre as séries de 1,2 e 3 minutos, e a PSE foi tomada de acordo com a escala de Borg (CR-10) a cada repetição. Os resultados não demonstraram diferenças significativa da PSE em relação ao tempo de intervalo. Em contrapartida, um estudo realizado por SiLva et al. ${ }^{25}$ demonstrou que a PSE obteve diferenças significativas de acordo com a diminuição do IR e o aumento dos níveis de fadiga. No entanto, a amostra deste estudo foi composta por adolescentes, o que diferencia de nossa investigação, mas proporcionando resultados similares. Já no estudo de SimẤo et al. ${ }^{27}$ os resultados mostraram que a PSE não sofreu alteração ao ser utilizada em 2 diferentes intervalos de tempo quando realizadas repetições máximas por homens adultos. É interessante verificar que nesse estudo o tempo de recuperação de intervalo entre as séries (2 e 5 minutos) não foi suficiente para alterar a PSE dos indivíduos, resultado diferente do encontrado em nosso estudo, isso pode se dever ao diferente tempo de intervalo, pois maiores tempos provocam uma recuperação mais completa e a percepção de esforço se mantem mais estável.

Os métodos de avaliação da PSE propostos pela literatura ao longo dos anos nos mostra ser uma importante estratégia para mensurar os níveis de fadiga e a intensidade do esforço de diferentes indivíduos ${ }^{29,30}$. Além de se revelar um método eficaz, de baixo custo e fácil acessibilidade ${ }^{31}$, a PSE vem sendo cada vez mais utilizada para prescrição de ERs, principalmente após a criação e validação da escala de OMNI-RES, desenvolvida para se obter o esforço físico no treinamento de força. Contudo, a utilização da mesma para controle de intensidade para homens e mulheres, ainda é questionável, necessitando de mais estudos acerca do tema.

O PSE avaliada através da escala OMNI-RES e o volume de repetições para diferentes intervalos de recuperação entre as séries de um exercício resistido realizado no aparelho Leg Press $45^{\circ}$ mostrou náo sofrer alteraçóes entre homens e mulheres fisicamente ativos. No entanto, aumentos significativos são apresentados na PSE entre as séries de ERs, independente de um IR de 1 ou 3 minutos em ambos os gêneros.

Os dados deste estudo demonstraram que apesar de não haver diferença entre os gêneros, IRs maiores estão diretamente relacionados a um maior volume de repetições em homens e mulheres fisicamente ativos.

\section{Abstract}

\section{Effect of different rest intervals in perceived exertion in men and women}

The time interval between sets in physical exercise is a variable that has been used as a means of controlling the intensity and density of training. The objective of the study was to investigate the Subjective Perception Effort (PSE) in intervals (RIs) of 1 and 3 min for 3 consecutive series in the apparatus Leg Press 45 to momentary concentric failure between men and women, using the OMNI - RES scale. Study participants were 14 volunteers, 7 men ( 22 years $\pm 3.14,78 \pm 11.42 \mathrm{~kg}, 1.74 \pm 0.05 \mathrm{~m}$ ) and 7 women ( 23 years \pm 3.76 , $56.4 \pm 6.88 \mathrm{~kg}, 1.63 \pm 0.07 \mathrm{~m}$ ), both physically active. Data were collected on alternate days and randomized manner, respecting an interval of 48 hours for blood sampling of the PSE. To prove the sample distribution the Shapiro Wilk test was used. To identify the differences between the number of repetitions and the PSE between genders was adopted by the Mann - Whitney test. The results showed that the comparison between the genders in the PSE and the number of repetitions in the two intervals studied variables was not possible to identify significant differences between genders. This occurred only in the sum of the total number of repetitions of the male and female intervals between the two groups and compared within each group the number of repetitions of the series. There was a decrease in the number of repetitions in the two IRs, 
however, no significant difference in RPE between sets in both intervals. The use of the same for intensity control for men and women is still questionable, requiring further studies on the subject.

KeYwords: Subjective Perception of Effort; Rest Intervals; Number of Repetitions; Gender Difference.

\section{Referências}

1. Bottaro M, Machado S, Nogueira W, Scales R, Veloso J. Effect of high versus low-velocity resistance training on muscular fitness and functional performance in older men. Eur J Appl Physiol. 2007;99(3):257-64.

2. Hill-Haas S, Coutts A, Rowsell G, Dawson B. Variability of acute physiological responses and performance profiles of youth soccer players in small-sided games. J Sci Med Sport. 2008;11(5):487-90.

3. American College of Sports Medicine. American College of Sports Medicine position stand: progression models in resistance training for healthy adults. Med Sci Sports Exerc. 2009;41(3):687-708.

4. Ide BN, Leme TC, Lopes CR, Moreira A, Dechechi CJ, Sarraipa MF, et al. Time course of strength and power recovery after resistance training with different movement velocities. J Strength Cond Res. 2011;25(7):2025-33.

5. Rahimi R. Effect of different rest intervals on the exercise volume completed during squat bouts. J Sports Sci Med. 2005;4(4):361-6.

6. Willardson JM, Burkett LN. The effect of rest interval length on the sustainability of squat and bench press repetitions. J Strength Cond Res. 2006;20(2):400-3.

7. Ratamess NA, Falvo MJ, Mangine GT, Hoffman JR, Faigenbaum AD, Kang J. The effect of rest interval length on metabolic responses to the bench press exercise. Eur J Appl Physiol. 2007;100(1):1-17.

8. Hill-Haas S, Bishop D, Dawson B, Goodman C, Edge J. Effects of rest interval during high-repetition resistance training on strength, aerobic fitness, and repeated-sprint ability. J Sports Sci. 2007;25(6):619-28.

9. Bottaro M, Russo AF, Oliveira RJ. The effects of rest interval on quadriceps torque during an isokinetic testing protocol in elderly. J Sports Sci Med. 2005;4(3):285-90.

10. Parcell AC, Sawyer, RD, Tricoli, VA, Chinevere TD. Minimum rest period for strength recovery during a common isokinetic testing protocol. Med Sci Sports Exerc. 2002;34(6):1018-22.

11. Ernesto C, Bottaro M, Silva FM, Sales MPM, Celes R, Oliveira RJ. Effects of different rest interval on isokinetic muscle performance among older adults. Rev Bras Fisioter. 2009;13(1):65-72.

12. Borg G. Escalas de Borg para a dor e esforço percebido. São Paulo: Manole; 2000.

13. Robertson RJ, Goss FL, Rutkowski J, Lenz B, Dixon C, Timmer J, et al. Concurrent validation of the OMNI Perceived Exertion Scale for resistance exercise. Med Sci Sports Exerc. 2003;35(2):333-41.

14. Barquilha G, Ribeiro AS, da Silva DRP, Oliveira JC, Azevedo PHSM, Cyrino ES. Efeito de diferentes intervalos de recuperação sobre a resistência de força em indivíduos de ambos os sexos. Rev Educ Fís/UEM. 2013;24(2):261-8.

15. Hunter SK. Sex differences and mechanisms of task-specific muscle fatigue. Exerc Sport Sci Rev. 2009;37(3):113-22.

16. Weir JP, Wagner LL, Housh TJ. The effect of rest interval length on repeated maximal bench presses. J Strength Cond Res. 1994;8(1):58-60.

17. Kraemer WJ. A series of studies: the physiological basis for strength training in American football: fact over philosophy. J Strength Cond Res. 1997;11(3):131-42.

18. Larson Junior GD, Potteiger JA. A comparison of three different rest intervals between multiple squat bouts. J Strength Cond Res. 1997;11(2):115-8.

19. Bottaro M, Martins B, Gentil P, Wagner D. Effects of rest duration between sets of resistance training on acute hormonal responses in trained women. J Sci Med Sport. 2009;12(1):73-8.

20. Jackson AS, Pollock ML. Generalized equations for predicting body density of men. British Journal Nutrition. 1978;40(3):497504.

21. Fermino RC, Winiarski ZH, Rosa RJ, Lorenci LG, Buso S, Simão R. Influência do aquecimento específico e de alongamento no desempenho da força muscular em 10 repetiçóes máximas. Rev Bras Ciênc Mov. 2005;13(4):25-32.

22. Chagas MH, Barbosa JRM, Lima FV. Comparação do número máximo de repetiçóes realizadas a 40 e $80 \%$ de uma repetição máxima em dois diferentes exercícios na musculação entre os gêneros masculino e feminino. Rev Bras Educ Fís Esporte. 2005;19(1):5-12. 
23. Haskell WL, Lee I, Pate RR, Powell KE, Blair SN, Franklin BA, et al. Physical activity and public health readapted recommendation for adults from the American College of Sports Medicine and the American Heart Association. Circulation. 2007;116:1081-93.

24. Baechle TR, Earle RW. Essentials of strength training and conditioning. Champaign: Human Kinetics; 2000.

25. Silva MS, Silva TS, Mota MR, Damasceno VO, Silva FM. Análise do efeito de diferentes intensidades e intervalos de recuperação na percepção subjetiva de atletas. Motricidade. 2011;7(1):3-12.

26. Kraemer WJ, Häkkinen K. Treinamento de força para o esporte. Porto Alegre: Artmed; 2004.

27. Simão R, Monteiro W, Jacometo A, Tesseroli C, Teixeira G. A influência de três diferentes intervalos de recuperação entre séries com cargas para 10 repetiçóes máximas. Rev Bras Ciênc Mov. 2006;14(3):37-44.

28. Woods S, Bridge T, Nelson D, Risse K, Pincivero DM. The effects of rest interval length on ratings of perceived exertion during dynamic knee extension exercise. J Strength Cond Res. 2004;18(3):540-5.

29. Pfeiffer KA, Pivarnik JM, Womack CJ, Reeves MJ, Malina RM. Reability and validity of the Borg and OMNI rating of perceived exertion scales in adolescent girls. Med Sci Sports Exerc. 2004;34(12):2057-61.

30. Rukowski JJ, Robertson RJ, Tseh WD, Caputo JL, Keefer DJ, Sutika KM, Morgan DW. Assessment of RPE signal dominance at slow-to moderate walking speeds in children using the OMNI perceived exertion scale. Pediatric Exercise Science. 2004;16(4):334-42.

31. Salles BF, Miranda F, Novaes J, Simão R. Influência de dois e cinco minutos de intervalo entre séries em exercícios mono e multiarticulares para membros inferiores. Remefe. 2008;7(1):35-44.

\begin{tabular}{r|c} 
ENDEREÇo & \\
Sandro Fernandes da Silva & \\
Departamento de Educação Física da & Recebido para publicação: 13/04/2014 \\
Universidade Federal de Lavras & Revisado: 28/10/2014 \\
Caixa Postal 3037 - Campus Universitário & Aceito: 10/o6/2015 \\
37200-000 - Lavras - MG - BRASIL & \\
e-mail: sandrofs@def.ufla.br & \\
&
\end{tabular}

